\title{
A MARGINAL DIMENSION OF A WEAKLY DILUTED QUENCHED $m$-VECTOR MODEL
}

\author{
Yu. Holovatch ${ }^{1,2}$, M. Dudka ${ }^{1}$, T. Yavors'kii ${ }^{2}$ \\ ${ }^{1}$ Institute for Condensed Matter Physics of the National Academy of Sciences of Ukraine \\ 1 Svientsitskii Str., Lviv, UA-79011, Ukraine \\ ${ }^{2}$ Ivan Franko National University of Lviv, Department for Theoretical Physics \\ 12 Drahomanov Str., Lviv, UA-79005, Ukraine
}

(Received August 31, 2001)

\begin{abstract}
We calculate a marginal order parameter dimension $m_{c}$ which in a weakly diluted quenched $m$ vector model controls the crossover from a universality class of a "pure" model $\left(m>m_{c}\right)$ to a new universality class $\left(m<m_{c}\right)$. Exploiting the Harris criterion and the field-theoretical renormalization group approach allows us to obtain $m_{c}$ as a five-loop $\varepsilon$-expansion as well as a six-loop pseudo- $\varepsilon$ expansion. In order to estimate the numerical value of $m_{c}$ we process the series by precisely adjusted Padé-Borel-Leroy resummation procedures. Our final result $m_{c}=1.912 \pm 0.004<2$ stems from the longer and more reliable pseudo- $\varepsilon$ expansion, suggesting that a weak quenched disorder does not change the values of $x y$-model critical exponents as it follows from the experiments on critical properties of $\mathrm{He}^{4}$ in porous media.
\end{abstract}

Key words: quenched disorder, $m$-vector model, renormalization group.

PACS number(s): 05.50.+q, 64.60.Ak, 75.10.Hk

\section{INTRODUCTION}

Along with the space dimension $d$, the order parameter dimension $m$ is relevant for the universal properties of a model at criticality [1]. In the presence of disorder the value of $m$ determines also whether a disordered model possesses novel universal properties in comparison with a pure model. A typical example of this feature is presented by a critical behaviour of a weakly diluted quenched $m$-vector model [2]. The model is defined by a Hamiltonian

$$
H=-\frac{1}{2} \sum_{i, j} J\left(\left|\mathbf{R}_{i}-\mathbf{R}_{j}\right|\right) \vec{S}_{\mathbf{R}_{i}} \vec{S}_{\mathbf{R}_{i}} c_{\mathbf{R}_{i}} c_{\mathbf{R}_{j}},
$$

where $\mathbf{R}_{i}$ span over the sites of a simple cubic lattice and $\vec{S}_{\mathbf{R}_{i}}$ denote the $m$-component spins interacting via a translationally invariant short-range isotropic interaction $J\left(\left|\mathbf{R}_{i}-\mathbf{R}_{j}\right|\right)$. The weak disorder is introduced by stochastic uncorrelated occupation numbers $c_{\mathbf{R}_{i}}$ equal to 1 in the case when a site is occupied by a magnetic atom and 0 otherwise (see Fig. 1). The concentration of occupied sites is considered to be above the percolation threshold. The quenched disorder implies that vacancies $c_{\mathbf{R}_{i}}=0$ are fixed and require configurational averaging of observables [3].

The crucial dependence of the universality class of model (1) on the order parameter dimension $m$ can be established by the Harris criterion [4]. It states that a disorder changes the universal critical properties of a "pure" model only if heat capacity critical exponent of a pure model is positive. Within the hierarchy of the physical realizations of the $m$-vector model only the Ising model $m=1$ is characterised by $\alpha=0.109 \pm 0.004>0$, while $x y-(m=2)$ and Heisenberg $(m=3)$ models' heat capacity does not exhibit divergency at critical- ity: the corresponding critical exponents remain negative $\alpha=-0.011 \pm 0.004, \alpha=-0.122 \pm 0.009$ [5]. Therefore one can expect that only weakly diluted quenched Ising model belongs to a new universality class.

Indeed, experimental studies confirm the theoretical prediction. The bulk of evidence collected in a recent review [6] demonstrate a novel critical behaviour of the magnetic systems described by model (1) at $m=1$. The experimental value of the heat capacity critical exponent at the $\lambda$-transition in He-4 [7] corroborated that the system belongs to the $x y$-model universality class with no divergency of the heat capacity at the phase transition point. Subsequently, experiments on the critical behaviour of He-4 in porous media [8] confirmed the irrelevancy of a weak quenched disorder in this case.

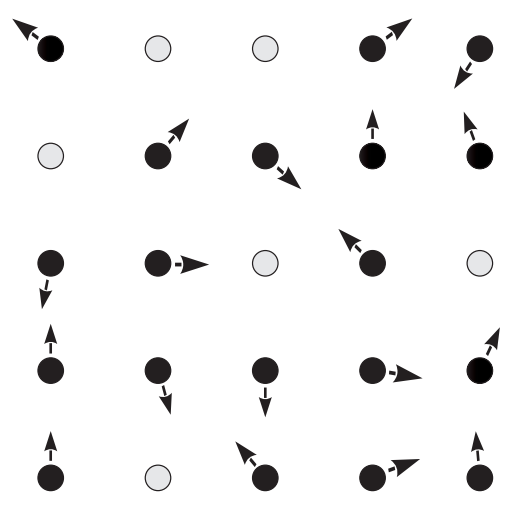

Fig. 1. The weakly diluted quenched $m$-vector model describes a system of randomly distributed $m$-component vectors which are fixed on sites of a three-dimensional cubic lattice and interact via a short-range translationally-invariant force. 
A natural question arises: can a marginal value of $m$ be obtained such that for $m>m_{c}$ the critical exponents of a weakly diluted quenched $m$-vector model coincide with the corresponding values of a "pure" model, but for $m<m_{c}$ they split to new values. Note, that while the values of the critical exponents both for pure [5] and diluted $m$-vector [9] models are calculated precisely, the crossover between two universality classes has not been the subject of extended theoretical calculations. One can mention only the results for $m_{c}$ on the basis of two- [10] and three-loop renormalization group studies [11] of a weakly diluted $d$-dimensional $m$-vector model. The obtained values $m_{c}=2.01$ and $m_{c}=2.12$ contradict both experimental data [8] and the Harris criterion [4]. In contrast, an alternative estimate on the base of a refined by conformal mapping resummation of the six-loop renormalization group functions of the $m$-vector model explicitly yielded $m_{c}=1.942 \pm 0.026$ [12]. In this study we want to solve the inconsistency of the theoretical results [10-12] and to perform an independent determination of $m_{c}$.

Relying on the Harris criterion one can determine $m_{c}$ from the requirement of vanishing the heat capacity critical exponent $\alpha$ of the $m$-vector model. We will make use of this condition appealing to a well-established field-theoretical renormalization group (RG) approach [1] which allows perturbative calculating of the marginal order parameter dimension $m_{c}$. In the next Section we obtain $m_{c}$ as $\varepsilon$ - and pseudo- $\varepsilon$ expansions which follow from the alternative minimal subtraction and massive schemes of field-theoretical renormalization group approach. In order to cope with the divergencies of the expansions and to obtain reliable numerical values based on them we process the expansions by appropriate resummation procedures. The resummation procedures are also analyzed and adjusted in the next Section. The outcomes of the study are presented in the concluding Section III.

\section{THE EXPANSIONS AND THE RESUMMATION}

As mentioned above, the marginal dimension $m_{c}$ of a weakly diluted $m$-vector model can be reconstituted from the critical properties of a "pure" $m$-vector model. Considering the heat capacity critical exponent $\alpha$ of a "pure" model as a function of the order parameter component number $m$, one can write the master equation for determining $m_{c}$ as follows:

$$
\alpha\left(m_{c}\right)=0
$$

The treatment of Eq. (2) by means of the field-theoretical $\mathrm{RG}$ approach can be performed in various schemes. Two of them are most widely used in the theory of critical phenomena. The dimensional regularisation with the minimal subtraction [13] allows obtaining quantities of interest by familiar $\varepsilon$-expansions [14] with $\varepsilon=4-d$. As a starting point to obtain the $\varepsilon$-expansion for $m_{c}$ serves the $\varepsilon$-expansion for the critical exponent $\alpha$ of the $m$-vector model, which is known in the five-loop the approximation [15]. By keeping the coefficients of the expansions as functions of $m$ and reexpanding equation (2) in $\varepsilon$ we obtain $m_{c}$ in the form:

$$
\begin{aligned}
m_{c}= & 4-4 \varepsilon+4.707199 \varepsilon^{2}-8.727517 \varepsilon^{3} \\
& +20.878373 \varepsilon^{4}
\end{aligned}
$$

Formally, the numerical value of $m_{c}$ at $d=3$ can be calculated from the expansion (3) by the substitution $\varepsilon=1$.

The renormalization conditions of the $R G$ massive scheme [16] provide another possibility to obtain the critical properties directly at $d=3$. The traditional calculation of the critical exponents in the massive field theoretical RG scheme implies a numerical analysis of the RG functions. However, the most accurate estimates of the critical exponents of a three-dimensional $m$-vector model $[5,17]$ are based on a pseudo- $\varepsilon$ expansion technique [18]. This technique avoids intrinsic errors accumulation typical for the numerical processing of the massive RG functions and results in a self-consistent collection of contributions from the different steps of calculations [17]. On the other hand, introducting the auxiliary pseudo$\varepsilon$ parameter $\tau$ which mimics the role of the ordinary $\varepsilon$ parameter of the minimal subtraction RG scheme allows to analyse the series by methods well-established for the $\varepsilon$-expansion.

In order to obtain a pseudo- $\varepsilon$ expansion for $m_{c}$ we start from the expansions of RG functions of $m$-vector model which within the massive scheme have been calculated in the six-loop approximation [19]. Introducing into functions [19] the parameter $\tau$ (see [17] for details) we obtain the pseudo- $\varepsilon$ expansion for $m_{c}$ at $d=3$ as follows:

$$
\begin{aligned}
m_{c}= & 4-8 / 3 \tau+0.766489 \tau^{2}-0.293632 \tau^{3} \\
& +0.193141 \tau^{4}-0.192714 \tau^{5}
\end{aligned}
$$

Again, the resulting numerical value of $m_{c}$ can be obtained from expansion (4) by the final substitution $\tau=1$.

The explicit form of expansions (3) and (4) is sufficient in principle to estimate the numerical value of $m_{c}$. However, the series for RG functions are known to be of asymptotic nature [20-22] and must be resummed before the final substitutions $\varepsilon=1(\tau=1)$. An explicit form of the asymptotic of series (3)-(4) has not been obtained, contrary to $\varepsilon$-expansions for the $m$-vector model critical exponents. Consequently, the applicability of resummation procedures to the series of $m_{c}$ is only conjectured.

A resummation procedure, which in different modifications is commonly used in the studies of asymptotic series, is the integral Borel transformation [23]. However, this technique implies explicit knowledge of the general term of a series and thus cannot be applied here, where only truncated sums of the series are known. On the other hand neither can we use the resummation proce- 
dures based on the conformal mapping technique [17] since even estimates on large-order order behaviour of expansions terms are unavailable. In the case of the series (3)-(4) we are restricted to the simplest procedures which do not imply such estimates.

Let us start the analysis by representing series (3) by means of Padé approximants $[M / N](x)=$ $\sum_{i=0}^{M} a_{i} x^{i} / \sum_{j=0}^{N} b_{j} x^{j}$ in the variable $x=\varepsilon$ [24]. The result for $m_{c}$ is shown in the form of a Padé table (5), where the number of the row, $M$, and that of the column, $N$, correspond to the order of the numerator and that of the denominator of the Padé approximant $[M / N]$ respectively. Subsequently, o denotes approximants which can not be constructed within the considered approximation, while small numbers in (5) indicate that approximants have poles (at $\varepsilon=6.52$ and $\varepsilon=1.11$ for $[0 / 2]$ and $[0 / 4]$ respectively) and thus are unreliable.

$$
\left[\begin{array}{ccccc}
4 & 2 & 2.1939 & 1.5086 & 6.1528 \\
0 & 2.1624 & 2.0316 & 1.9365 & o \\
4.7072 & 1.6493 & 1.9208 & o & o \\
-4.0203 & 2.1344 & o & o & o \\
16.858 & o & o & o & o
\end{array}\right]
$$

The first column of table (5) represents a straightforward summation of series (3) terms and obviously shows the divergence of results with the increase of the approximation order. Conversely, the convergence of numbers is observed along the main and next to main diagonals of the table. For instance, the expected inequality $m_{c}<2$ can be obtained already within the fourth and the fifth order of the $\varepsilon$-expansion (3).

The results of Padé-analysis of the pseudo- $\varepsilon$ expansion (4) are presented in table (6) in the same notations as in table (5).

$\left[\begin{array}{cccccc}4 & 2.4 & 2.0839 & 1.9669 & 1.9398 & 1.9106 \\ 1.3333 & 1.9287 & 1.8799 & 1.9311 & 2.2425 & o \\ 2.0998 & 1.8875 & 1.9084 & 1.9085 & o & o \\ 1.8062 & 1.9227 & 1.9085 & o & o & o \\ 1.9993 & 1.9029 & o & o & o & o \\ 1.8066 & o & o & o & o & o\end{array}\right]$

Again, small numbers correspond to unreliable approximants with the poles at $\tau=7.29$ and $\tau=0.907$ for $[1 / 2]$ and $[1 / 4]$ respectively. The distinguishing property of table (5) is a convergence of the results on the basis of a mere summation of the pseudo- $\varepsilon$ expansion terms (4) (the first column of table (6)). Contrary to the $\varepsilon$ expansion analysis (5), the inequality $m_{c}<2$ is observed already in a four-loop approximation and remains valid in the five- and the six-loop approximations. However, the best convergence is noticed for the approximants parallel to the main diagonal of table (6). For instance, the six-loop approximants $[3 / 2]$ and $[2 / 3]$ as well as the fiveloop approximant $[2 / 2]$ yield practically the same value of $m_{c}$ within the fourth digit leading to the estimate $m_{c}=1.9085$. This number is not yet considered as the most accurate estimate of $m_{c}$ that one can obtain from expansion (4).

By assuming the factorial divergence of the coefficients of expansions (3), (4), one can apply to their analysis a refined Padé-Borel-Leroy resummation procedure which has been successfully used in various tasks of theory of critical phenomena [25]. The procedure is based on the integral Borel transformation [23], however it uses as an intermediate step an extrapolation by means of a Padéapproximant [24]. More precisely, the procedure is defined by the following algorithm:

- starting from the initial sum $S$ of $L$ terms one constructs its Borel-Leroy image

$$
S(x)=\sum_{i=0}^{L} a_{i} x^{i} \Rightarrow S^{\mathrm{B}}(x t)=\sum_{i=0}^{L} \frac{a_{i}(x t)^{i}}{(i+b) !}
$$

where $b$ is an arbitrary non-negative number;

- subsequently one extrapolates the Borel-Leroy image $(7)$ by a rational Padé approximant

$$
S^{\mathrm{B}}(x t) \quad \Rightarrow \quad[M / N](x t)
$$

- the resummed function $S^{\text {Res }}$ is finally obtained in the following form:

$$
S^{\operatorname{Res}}(x)=\int_{0}^{\infty} d t \exp (-t) t^{b}[M / N](x t)
$$

Similar to the Padé-analysis, within the Padé-BorelLeroy resummation procedure, various final estimates of a resummed series can be obtained depending on the type of the Padé approximants chosen. In addition the fit parameter $b$ can be used for adjusting the resummation procedure to provide a self-consistent convergence of the results. In Fig. 2 we present the result of the $\varepsilon$-expansion processing by means of the resummation procedure $(7)-$ (8). Here, the estimates of $m_{c}$ on the basis of the higher three-, four- and five-loop $\varepsilon$-expansion are depicted depending on the approximant type as well as on the fit parameter $b$. Among all the possibilities we chose the approximants close to the main diagonal, namely $[1 / 1]$, $[0 / 2]$ in three-, $[2 / 1],[1 / 2]$ in four and $[3 / 1],[2 / 2],[1 / 3]$ in the five-loop approximation. Notably, approximants having poles on the real positive semiaxis are considered as unreliable. For instance, Fig. 2 displays the real part of values with imaginary parts smaller than $10^{-7}$.

Since no information is available on the large-order behaviour of series (3), all the values of $b$ can be considered as equally suitable. An evident property of all the curves in Fig. 2 is a saturation of the value of $m_{c}$ for large values of $b$. In order to exclude the values on the basis of 
large $b$ and taking into account that in similar tasks of critical phenomena $b$ accounts for several units, we restrict ourselves only to the values of $b$ within the interval $0 \leq b \leq 5$. We neither take into account the approximant $[\overline{1 / 2}]$ since it provides only two values within the interval.

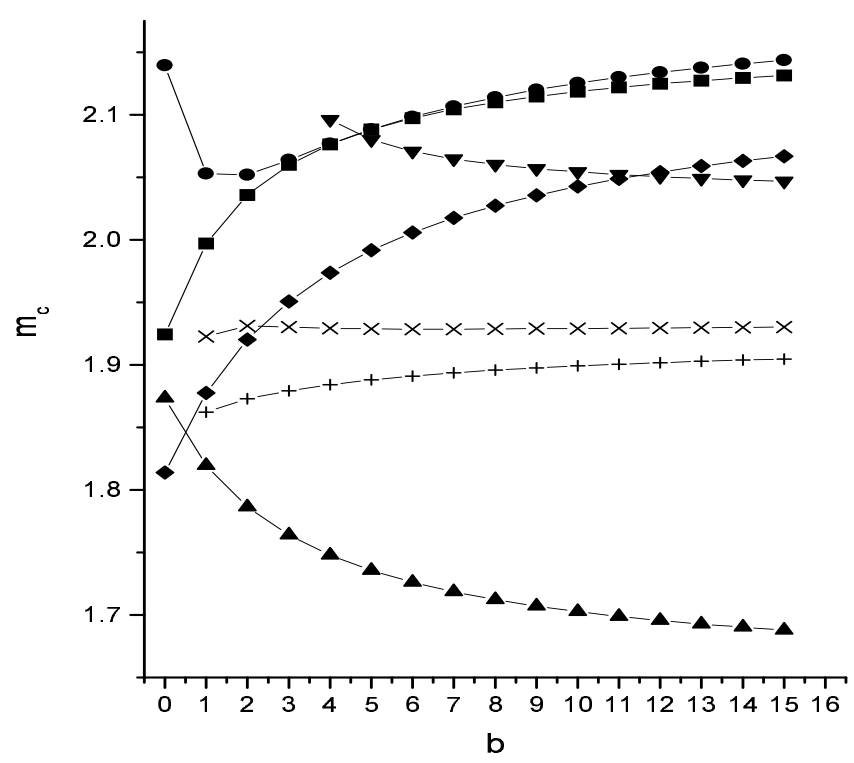

Fig. 2. The estimate of the marginal order parameter dimension $m_{c}$ obtained on the basis of Padé-Borel-Leroy resummation of the $\varepsilon$-expansion (3) for different values of the fit parameter $b$. The following symbols show the results based on different Padé approximants: $\square$ and $\bullet$ mean the [1/1], [0/2] approximants, $\boldsymbol{\Delta}$ and $\mathbf{\nabla}$ show [2/1] and [1/2] approximants, while $\downarrow+$ and $\times$ denote the $[3 / 1],[2 / 2]$ and $[1 / 3]$ approximants respectively.

To obtain a confidence interval for $m_{c}$ we analyse the data of Fig. 2 in the following manner. As a first step we obtain the central value of $m_{c}$ on falling look on the data on each approximant separately. To this end we average all the values on the basis of each approximant over $b, 0 \leq b \leq 5$. The corresponding error bars are then determined by a half of the difference between the maximal and minimal values of $m_{c}$ within the considered interval of $b$. The resulting estimates of $m_{c}$ in the successive number of loops are presented in Fig. 4, a, where the result on the basis of each working approximant is shown by its central value (black circles) and the error bars. For instance, in the three-loop approximation two confidence intervals correspond to $[1 / 1]$ (a smaller central value) and $[0 / 2]$ (a larger central value) approximants; the four-loop approximation contributes by a single estimate on the basis of [2/1] approximant, while the five-loop approximation yields three confidence intervals from the $[1 / 3]$ (the largest central value), [3/1] (the middle central value) and [2/2] approximants.

In order to get a final estimate of $m_{c}$ within each or- der of perturbation theory we take up the values on the basis of different approximants of the same order as independent. Doing so allows us to consider their average as an overall estimate of $m_{c}$ within a given number of loops. The overall estimates of $m_{c}$ obtained in this way depend on the loop order oscillatively, which permits to suppose that a successive central value lies between two preceding central values. Thus we average the overall estimate on the basis of the approximation order with a corresponding overall estimate from the preceding order, choosing the error bars as a half of a difference between maximal and minimal values from these pairs. Finally, we obtain a sequence of confidence intervals of $m_{c}$ such that error bars of a higher result lie completely within error bars of the previous one.

Based on the analysis described above we obtain the following estimates of $m_{c}$ from the $\varepsilon$-expansion (3):

$$
\begin{aligned}
& \text { 4LA }: \quad m_{c}=1.996 \pm 0.104, \\
& 5 \mathrm{LA}: \quad m_{c}=1.923 \pm 0.051 .
\end{aligned}
$$

One can note from estimates (9) that only the five-loop $\varepsilon$-expansion excludes the values $m_{c} \geq 2$, and therefore brings about a weakly quenched disorder irrelevancy for the $x y$-model universality class.

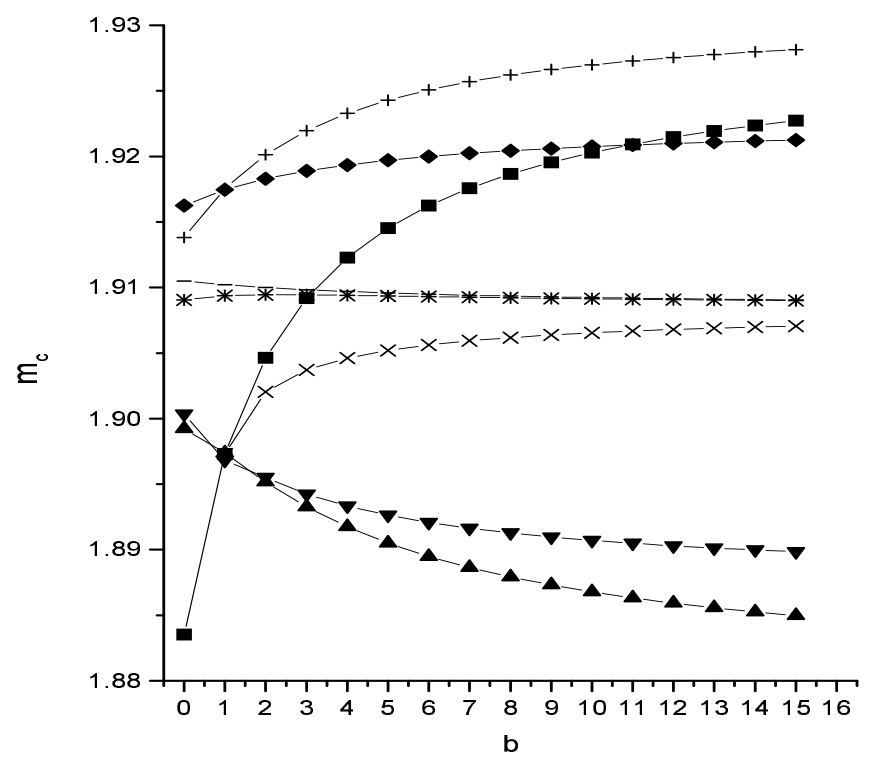

Fig. 3. The estimate of the marginal order parameter dimension $m_{c}$ obtained on the basis of Padé-Borel-Leroy resummation of the pseudo- $\varepsilon$ expansion (4) for different values of the fit parameter $b$. The following symbols show the results based on different Padé approximants: $\mathbf{\square}, \mathbf{\Lambda}, \mathbf{\nabla}$ denote the results on the base of [1/1], [2/1], [1/2] approximants, $\downarrow,+$ and $x$ show the $[3 / 1],[1 / 3],[2 / 2]$ approximants, while $*$ and correspond to $[2 / 3]$ and $[3 / 2]$ approximants respectively.

Let us turn now to the Padé-Borel-Leroy analysis of the pseudo- $\varepsilon$ expansion (4). The dependencies of the estimates of $m_{c}$ on the basis of the pseudo- $\varepsilon$ expansion on 
the fit parameter $b$ are shown for different approximants types increasing number of loops from three to six in Fig. 3. Since the pseudo- $\varepsilon$ expansion is one order longer, in addition to the approximants of Fig. 2 we use here the approximants of the six-loop order, namely the neardiagonal approximants $[3 / 2]$ and $[2 / 3]$. The values of $m_{c}$ obtained on the basis of the approximant $[0 / 2]$ do not fit Fig. 3 and thus are not presented, though they will be taken into account in the final calculations.

Applying the procedure identical to the $\varepsilon$-expansion we obtain the resulting estimates of $m_{c}$ depending on the type of the approximant as presented in Fig. 4, b. For instance, in the three-loop approximation two confidence intervals correspond to $[1 / 1]$ (smaller central value) and [0/2] (larger central value) approximants; the four-loop approximation contributes by two almost identical estimates on the basis of $[1 / 2]$ and [2/1] approximants, while the five-loop and the six-loop approximations yield three and two confidence intervals respectively. Subsequently, averaging the overall estimate on the basis of a given loop order with the corresponding overall estimate from a preceding order, one obtains a sequence of convergent confidence intervals for $m_{c}$ :

$$
\begin{array}{ll}
4 \mathrm{LA}: & m_{c}=1.976 \pm 0.117 \\
5 \mathrm{LA}: & m_{c}=1.904 \pm 0.013 \\
6 \mathrm{LA}: & m_{c}=1.912 \pm 0.004
\end{array}
$$

The sequence of results (10) obtained on the basis of the pseudo- $\varepsilon$ expansion (4) shows evidently better convergence properties than the corresponding results (9) obtained on the basis of the $\varepsilon$-expansion (3). For instance, the error bar of the five-loop $\varepsilon$-expansion estimate is four times larger than the corresponding estimate on the basis of pseudo- $\varepsilon$.
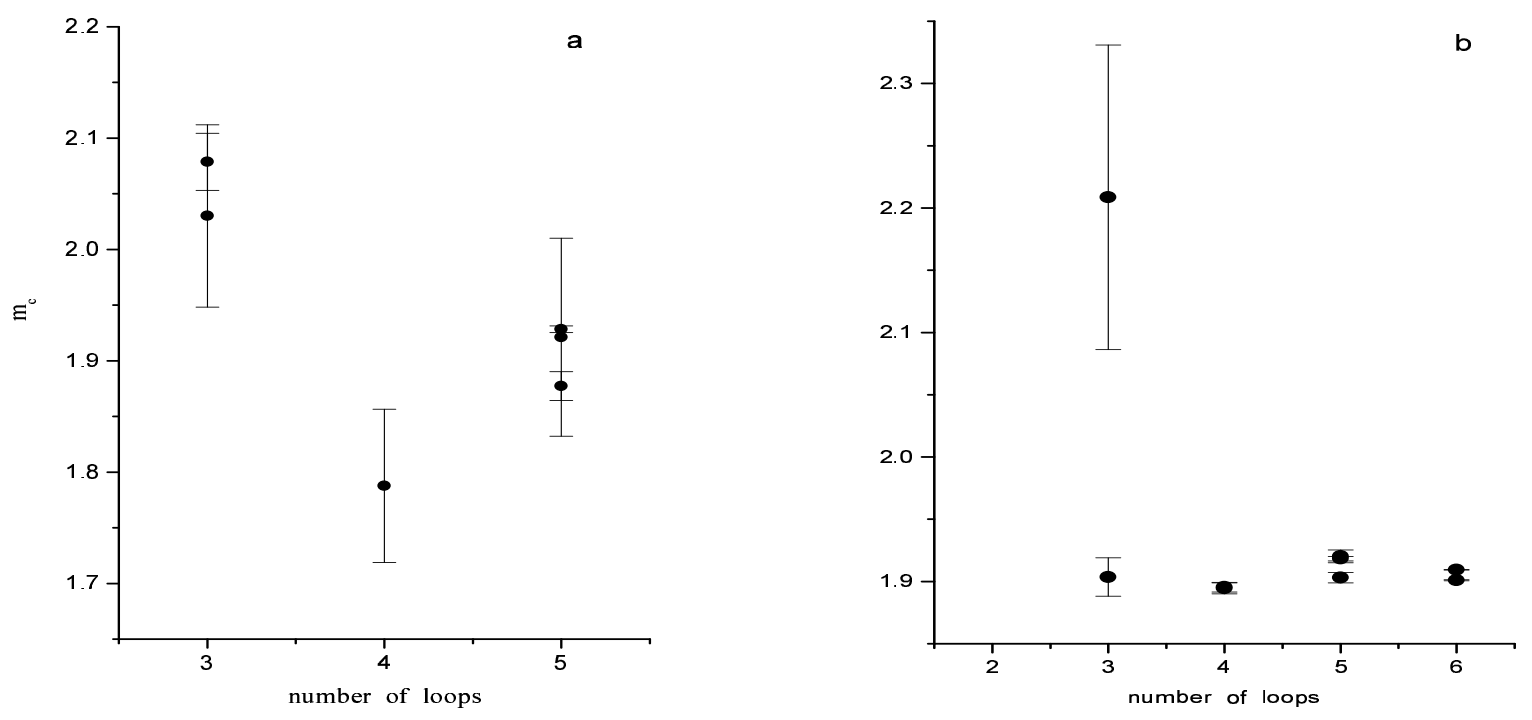

Fig. 4. The confidence intervals for the marginal order parameter dimension $m_{c}$ of a weakly diluted $m$-vector model as functions of the order of approximation. The left picture presents the result of the Padé-Borel-Leroy resummation of the $\varepsilon$-expansion (3), the right-hand picture corresponds to the analysis of the pseudo- $\varepsilon$ expansion (4). See the text for the whole description.

\section{CONCLUSIONS}

In the theory of critical phenomena the values of critical exponents and critical amplitudes ratios have attracted considerable interest of researchers. This study, however, has calculated another important quantity, which is responsible for a crossover phenomenon in the weakly diluted quenched $m$-vector model. Based on the Harris criterion as well as on the field-theoretical renormalization group approach we determined the marginal order parameter dimension $m_{c}$ of the model. Before our work only one reliable numerical estimate of $m_{c}$ was ob- tained [12]. The result $m_{c}=1.942 \pm 0.026$ [12] stemmed from the application of a conformal mapping resummation technique to the massive six-loop RG functions at $d=3$. We completed this study with two more results from two different RG schemes. Within the minimal subtraction scheme $m_{c}$ is obtained as an $\varepsilon$-expansion (3), while within the massive scheme $m_{c}$ is calculated as a pseudo- $\varepsilon$ expansion (4). Both series allow to estimate a numerical value of $m_{c}$ after their analysis by PadéBorel-Leroy resummation procedure, however the final estimate of the paper $m_{c}=1.912 \pm 0.004$ is based on the pseudo- $\varepsilon$ expansion. This is explained not only by 


\title{
YU. HOLOVATCH, M. DUDKA, T. YAVORS'KII
}

a longer series calculated for the expansion, but also by its better convergent properties in comparison with the $\varepsilon$-expansion. Such a situation has been already observed for a calculation of a marginal dimension of a cubic model [26].

Though the obtained value of $m_{c}$ is very close to the integer value 2 , our estimate evidently shows that within the error bars it is slightly smaller than 2 . This result im- plies that universal properties of the $x y$-model are not affected by a weakly quenched disorder at criticality. This conclusion is confirmed by recent experimental studies on the critical behaviour of the superfluid $\mathrm{He}^{4}$ in porous media [8].

We thank Claude Bervillier for useful discussions and Ted Knoy for a thorough reading of the manuscript.
[1] See, e. g. D. J. Amit Field Theory, the Renormalization Group, and Critical Phenomena (World Scientist, Singapore, 1989).

[2] H. E. Stanley, Phys. Rev. Lett 20, 589 (1968).

[3] R. Brout, Phys. Rev. 115, 824 (1959).

[4] A. B. Harris, J. Phys. C 7, 1671 (1974).

[5] R. Guida, J. Zinn-Justin, J. Phys. A 31, 8103 (1998).

[6] R. Folk, Yu. Holovatch, T. Yavors'kii, preprint condmat/0106468 (2001), to appear in: Physics Uspekhi (2002).

[7] The original result $\alpha=-0.01285 \pm 0.00038$ presented in Ref. [J. A. Lipa, D. R. Swanson, J. A. Nissen, T. C. P. Chui, U. E. Israelsson, Phys. Rev. Lett. 76, 944 (1996)] is incorrect. However, a new estimate reported in Ref. [J. A. Lipa et al., Phys. Rev. Lett. 84, 4894, (2000)] also yields a negative value of the exponent: $\alpha=-0.01056 \pm 0.00038$.

[8] J. Yoon, M. H. W. Chan, Phys. Rev. Lett. 78, 4801 (1997); G. M. Zassenhaus, J. D. Reppy, Phys. Rev. Lett. 83, 4800 (1999).

[9] A. Pelissetto, E. Vicari, Phys. Rev. B 626393 (2000).

[10] J. Jug, Phys. Rev B 27, 609 (1983); Yu. Holovatch, M. Shpot, J. Stat. Phys. 66, 867 (1992).

[11] Yu. Holovatch, T. Yavors'kii, Cond. Mat. Phys. 11, 87 (1997); Yu. Holovatch, T. Yavors'kii, J. Stat. Phys. 92, 785 (1998).

[12] C. Bervillier, Phys. Rev. B 34, 8141 (1986).
[13] G.'t Hooft, M. Veltman, Nucl. Phys. B 44, 189 (1972); G.'t Hooft, Nucl. Phys. B 61, 455 (1973).

[14] K. G. Wilson, M. E. Fisher, Phys. Rev. Lett. 28, 240 (1972).

[15] H. Kleinert, J. Neu, V. Schulte-Frohlinde, K. G. Chetyrkin, S. A. Larin, Phys. Lett. B 272, 39 (1991); Erratum: Phys. Lett. B 319, 545 (1993).

[16] G. Parisi (1973), unpublished; G. Parisi, J. Stat. Phys. 23, 49 (1980).

[17] J. C. Le Guillou, J. Zinn-Justin, Phys. Rev. B 21, 3976 (1980).

[18] The pseudo- $\varepsilon$ expansion was introduced by B. G. Nickel, see citation 19 in Ref. [17].

[19] S. A. Antonenko, A. I. Sokolov, Phys. Rev. E 51, 1894 (1995).

[20] L. Lipatov, Sov. Phys. JETP 45, 216 (1977).

[21] E. Brézin, J. Le Guillou, J. Zinn-Justin, Phys. Rev. D 15, 1544 (1977).

[22] E. Brézin, G. Parisi, J. Stat. Phys. 19, 269 (1978).

[23] G. H. Hardy, Divergent Series (Oxford, 1948).

[24] G. A. Baker, Jn, P. Graves-Morris, Padé Approximants (Addison-Wesley: Reading, MA, 1981).

[25] G. A. Baker, B. G. Nickel, M. S. Green, D. I. Meiron, Phys. Rev. Lett. 36, 1351 (1976); G. A. Baker, B. G. Nickel, D. I. Meiron, Phys. Rev. B 171365 (1978).

[26] R. Folk, Yu. Holovatch, T. Yavors'kii Phys. Rev. B 62, 12195 (2000); Erratum: Phys. Rev. B 63, 189901 (2001)

\section{ГРАНИЧНА ВИМІРНІСТЬ СЛАБОРОЗВЕДЕНОЇ ЗАМОРОЖКНОӦ $m$-ВЕКТОРНОЇ МОДЕЛІ}

\author{
Ю. Головач ${ }^{1,2}$, М. Дудка ${ }^{1}$, Т. Яворський ${ }^{2}$ \\ ${ }^{1}$ Інститут фізики конденсованих систем НАН Украӥни \\ вул. Свєниіиького, 1, Львів, 79011, Украйна \\ 2 Львівсъкий національний університет імені Ібана Франка, кафедра теоретичной фізики \\ вул. Драгоманова, 12, Львів, 79005, Украӥна
}

\begin{abstract}
Критична поведінка слаборозведеної замороженої $m$-векторної моделі визначається ,двома різними наборами показників. При достатньо великих значеннях параметра впорядкування $m$ модель у критичному режимі не відчуває присутности безладу, тоді як при малих значеннях $m$ вона належить до нового класу універсальності. Кросовер між двома різними режимами відбувається при певному граничному значенні $m_{c}$. Згідно з критерієм Гаріса $m_{c}$ можна визначити з умови занулення критичного показника теплоємности $\alpha(m)$ “чистої" моделі. На сьогодні стало стандартним використовувати теоретико-польовий ренормалізапійно груповий пілхід як засіб отримання кількісного опису критичних явищ. Тоді як рядли теорії збурень для критичних показників моделі отримані і проаналізовані багатьма способами, існує тільки одна надійна
\end{abstract}


чисельна оцінка для $m_{c}$. Підставою для ощінки $m_{c}=1.942 \pm 0.026 \in$ пересумовування за допомогою конформного відображення шестипетлевих ренормалізапійно-групових функпй масивної схеми при вимірності простору $d=3$. У нашій роботі ми доповнили це дослідження ше двома результатами. Спершу ми використову$є м о \varepsilon=4-d$-розклад, який дає розбіжний ряд: $m_{c}=4.0-4.0 \varepsilon+4.707199 \varepsilon^{2}-8.727517 \varepsilon^{3}+20.878373 \varepsilon^{4}$. Далі ми дістаємо псевдо- $\varepsilon$ розклал при $d=3: m_{c}=4-2.666667 \tau+0.766489 \tau^{2}-0.293632 \tau^{3}+0.193141 \tau^{4}-0.192714 \tau^{5}$. Найточніша оңінка випливає зі псевдо- $\varepsilon$ ряду. Застосовуючи пересумовування Паде-Бореля-Лєруа для ві,дновлення збіжности пњого ряду, ми отримуємо $m_{c}=1.912 \pm 0.004$. 